\title{
Histiocytic sarcoma of the neck: A case report
}

\author{
JIAN ZHOU ${ }^{1,2^{*}}$, YUE LIU $^{3 *}$, WANCHUN WANG ${ }^{1}$ and FENG-LEI XU ${ }^{4}$ \\ ${ }^{1}$ Department of Orthopedics, The Second Xiangya Hospital, Central South University, Changsha, Hunan 410011; \\ ${ }^{2}$ Department of Sports Medicine Research Center, Central South University, Changsha, Hunan 410008; \\ ${ }^{3}$ Center for Reproductive Medicine, Shandong University, Jinan, Shandong 250012; ${ }^{4}$ Department of \\ Otorhinolaryngology, The Second Hospital of Shandong University, Jinan, Shandong 250000, P.R. China
}

Received January 15, 2018; Accepted April 24, 2018

DOI: $10.3892 / \mathrm{mco} .2018 .1617$

\begin{abstract}
Histiocytic sarcoma (HS) is a term used to describe malignant hyperplasia of cells exhibiting morphological and immunophenotypical characteristics similar to those of mature cells, with expression of one or more tissue cell markers, excluding acute monocytic leukemia and primitive monocytic sarcoma. We herein describe a case of histiocytic sarcoma of the neck supported by histopathological and immunohistological evidence. A 53-year-old female patient of Chinese descent presented with a rapidly enlarging right neck mass. Imaging studies revealed multiple right cervical lymphadenectases with right jugular vein involvement. The tumor was composed of diffusely distributed large non-cohesive tumor cells, round or oval and focally spindle-shaped. The tumor cells were immunopositive for macrophage-associated antigen CD68 and lysosomes, mostly consistent with a diagnosis of HS. HS is very prone to systemic metastasis; therefore, early diagnosis and timely treatment are crucial.
\end{abstract}

Correspondence to: Professor Wanchun Wang, Department of Orthopedics, The Second Xiangya Hospital, Central South University, 139 Renmin Middle Road, Changsha, Hunan 410011, P.R. China

E-mail: wanchun.wang@csu.edu.cn

Professor Feng-lei Xu, Department of Otorhinolaryngology, The Second Hospital of Shandong University, 247 Beiyuan Avenue, Jinan, Shandong 250000, P.R. China

E-mail: fengleixu1@outlook.com

*Contributed equally

Abbreviations: HS, histiocytic sarcoma; CD, cluster of differentiation; CT, computed tomography; CK, cytokeratin; ALK, anaplastic lymphoma kinase; EMA, epithelial membrane antigen; FDCS, follicular dendritic cell sarcoma

Key words: histiocytic sarcoma, neck, CD68, immunohistochemistry, malignant tumour

\section{Introduction}

Histiocytic sarcoma (HS) is a rare malignant neoplasm originating from the hematopoietic system, composed of cells exhibiting morphological and immunophenotypical characteristics of mature tissue histiocytes, first described in 1939 as histiocytic medullary reticulosis, and as malignant histiocytosis by Rappaport in 1966 (1). Current epidemiological data estimate that $<1 \%$ of tumors presenting in lymph nodes or soft tissue can be defined as HS (2). Approximately 1/3 of the cases have been reported to occur in lymph nodes, while the incidence of extranodular HS, such as in the gastrointestinal tract, spleen and soft tissue, is relatively low (3).

Definitive morphological diagnosis of HS is difficult, and it must be verified by immunohistochemistry. The tumor cells express one or more tissue cell antigens, including CD163, which is the most important antigen for detecting macrophages (4), CD68 and lysozyme.

The aim of the present report is to present in detail the clinical and immunophenotypical characteristics of a case of HS.

\section{Case report}

A 53-year-old female patient of Chinese descent presented with a 3-month history of a rapidly enlarging right neck mass. The mas was mobile and tender, and was associated with symptoms including hoarseness, pharyngeal pain and occasionally coughing. Imaging studies, including neck ultrasound and contrast-enhanced computed tomography (CT) revealed multiple right cervical lymphadenectases with right jugular vein involvement (Fig. 1A). Cervical biopsy of the lesion was performed, followed by hematoxylin and eosin staining for $30 \mathrm{~min}$ at $37^{\circ} \mathrm{C}$, which revealed enlarged lymph nodes around the anterior border of the sternocleidomastoid muscle, with a greatest diameter of $3 \mathrm{~cm}$, adhering to the surrounding tissue and fusing with deep cervical lymph nodes. Microscopic examination revealed diffusely distributed large non-cohesive tumor cells, round or oval and focally spindle-shaped, with abundant eosinophilic cytoplasm and large bizarre pleomorphic nuclei. Phagocytosis of red blood cells, multinucleated giant cells and nuclear mitosis were readily identified (Fig. 1B). Immunohistochemical examination (antibody dilution, 1:100; OriGene Technologies, Inc., Wuxi, China) was negative 


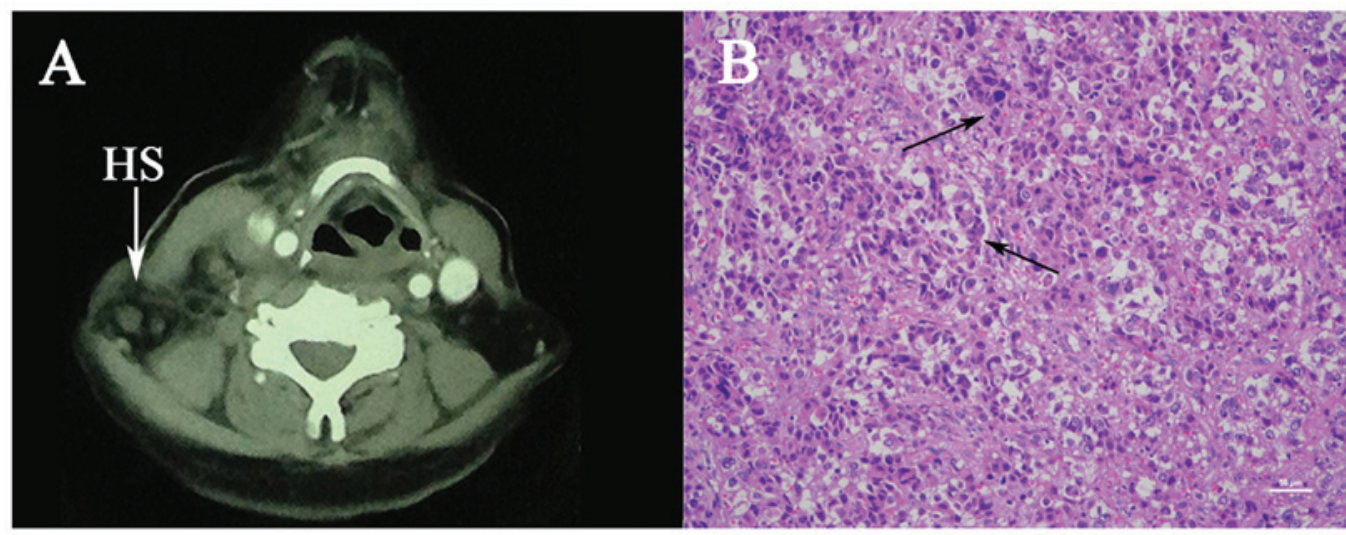

Figure 1. (A) Computed tomography of the neck showing multiple right cervical lymphadenectases. (B) Hematoxylin and eosin staining of the tumor (magnification, x20); arrows, HS cells. HS, histiocytic sarcoma.

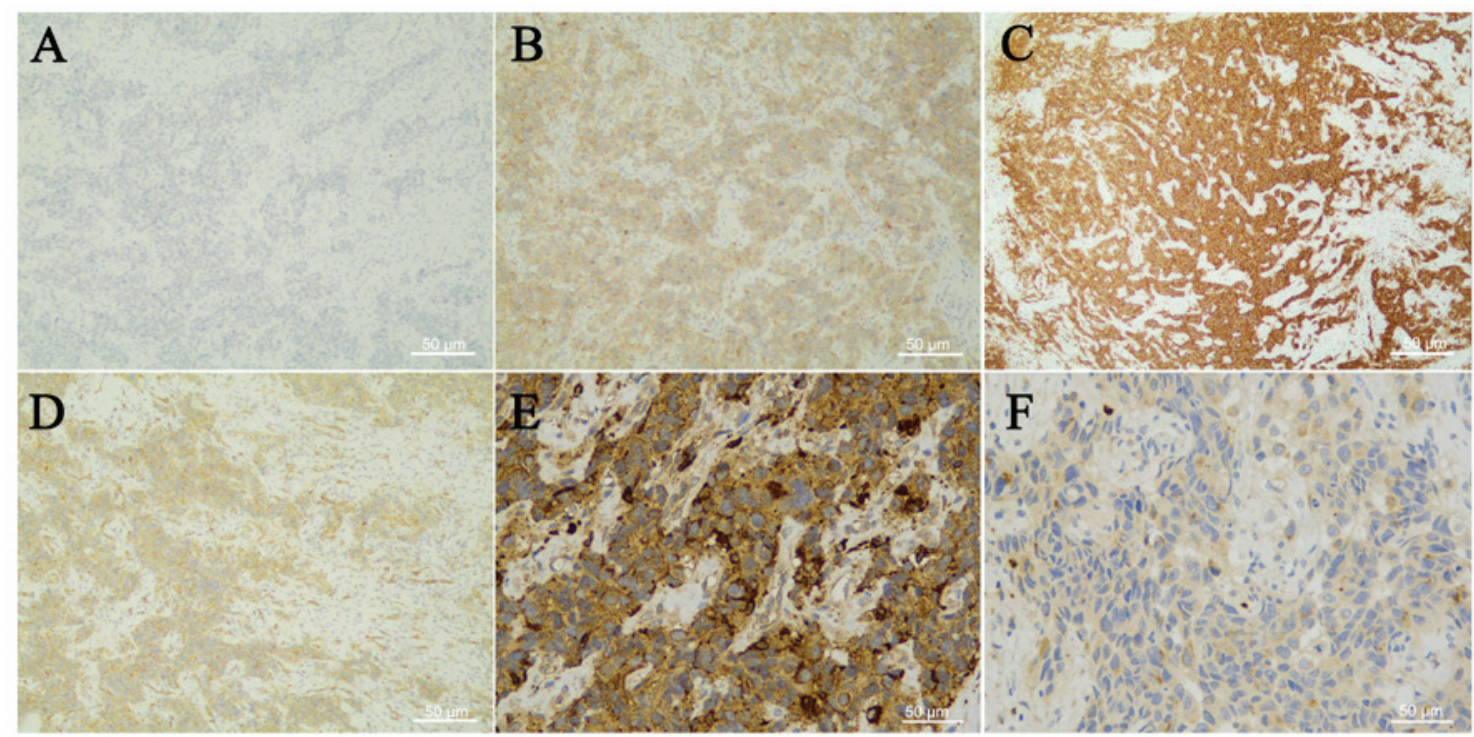

Figure 2. Immunohistochemical staining for (A) cytokeratin, (B) CD4, (C) CD5, (D) CD31, (E) CD68 and (F) lysozyme (magnification, x 100; scale bar, 1: 2,000).

for cytokeratin (CK; Fig. 2A), CD117, paired box (PAX)-5, S-100, CD20, anaplastic lymphoma kinase (ALK), CD34 and epithelial membrane antigen (EMA), ruling out epithelial, melanocytic or myeloid origin, while it was positive for CD4, CD5, CD31 (Fig. 2B-D) and vimentin, which complicated the diagnosis. However, the expression of histiocytic antigens, CD68 (Fig. 2E) and lysozyme (Fig. 2F), confirmed the diagnosis of HS (5), with a Ki-67 proliferation labeling index of $90 \%$. As it is a rare malignant neoplasm originating from the lympho-hematopoietic system, HS usually occurs in the lymph nodes and skin (6), it may affect multiple systems and carries a dismal prognosis. An enhanced CT of the chest, abdomen and pelvis revealed a right cervical space-occupying lesion and a nodular lesion in the superior lobe of the right lung, multiple mediastinal lymphadenectases, calcified lesions in the left lobe of the liver and fibroids. As there are currently no consensus guidelines for the management of HS due to its rarity, following an extensive discussion with several clinical departments, it was decided to commence chemoradiotherapy, as surgery would be associated with a high risk of incomplete clearance of the tumor and poor prognosis. However, the patient and her family asked to be discharged to recuperate prior to further treatment. The patient was discharged on July 15, 2016 and was lost to follow-up.

Due to its rapid invasiveness and high mortality rate, HS remains a medical challenge for which there is yet no effective treatment $(7,8)$, which calls for more efforts and further research.

\section{Discussion}

HS is an extremely rare lymphoid hematopoietic malignancy with a morbidity of $<0.5 \%$ among lymphoid hematopoietic tumors. In the 2016 revision of the World Health Organization classification of lymphoid neoplasms, HS is listed under the major categories of histiocyte and dendritic cell tumors, defined as malignant hyperplasia of cells exhibiting morphological and immunophenotypical characteristics similar to those of mature cells, with expression of one or more tissue cell markers, excluding acute monocytic leukemia and primitive monocytic sarcoma. HS may occur at any age, but the majority of the cases are encountered in adults, with a median age of 
52 years and a slight male predominance. Approximately $1 / 3$ of the cases occur in lymph nodes, while the incidence of extranodular HS, such as in the gastrointestinal tract, spleen and soft tissue, is relatively low.

Histologically, HS is characterized by destruction of normal tissue structure by diffuse, non-aggregated proliferative tumor cells, which appear as monoclonal or pleomorphic. The tumor cells are usually large, round or oval and focally spindle-shaped, with abundant eosinophilic cytoplasm surrounding large bizarre pleomorphic nuclei. Phagocytosis of red blood cells, multinucleated giant cells and nuclear mitoses were readily identified.

A definitive morphological diagnosis of HS is very difficult and it must be confirmed by immunohistochemistry. The tumor cells may express one or more tissue cell antigens, including CD163, CD68, lysozyme, CD11c and CD14, while CD45, leukocyte common antigen RO subtype, CD4, macrophage antibody 387 and $\alpha 1$-antichymotrypsin may also be positive in HS.

Langerhans cell marker (CD1a, langerin), myeloperoxidase (MPO), CD1, CD33, CD34, dendritic cell markers (CD21, CD35 etc.), specific B-cell and T-cell antigens, CD30, human melanoma black (HMB)-45 and CK are negative in HS. S-100 may be positive or negative. The Ki-67 proliferation index ranges from 10 to $90 \%$ (median, 20\%), but whether the percentage reflects the prognosis remains uncertain. Electron microscopy for the diagnosis of HS is of auxiliary value, and can demonstrate tumor cells with abundant cytoplasm, containing a large number of lysosomes, but without Birbeck bodies or intercellular junctions.

Histologically, HS may be misdiagnosed as several diseases: i) Large-cell lymphoma, mainly Hodgkin's lymphoma (expression of CD30, CD15, PAX-5), diffuse large B-cell lymphoma (expressing B cell markers) and anaplastic large-cell lymphoma (CD30, ALK and EMA positivity are considered as identification markers in addition to T-cell markers); ii) poorly differentiated carcinoma expresses epithelial markers such as CK and EMA; iii) malignant melanoma also expresses HMB-45 and melan A in addition to S-100; iv) granulocytic sarcoma is positive for the granulocyte-specific markers MPO and CD33, which may help in the differentiation from HS; v) follicular dendritic cell sarcoma (FDCS); the cells of FDCS are mostly spindle-shaped and oval with large nuclei, forming unequally sized nodules, and are positive for CD21, CD23 and CD35, while they are negative for CD68.

HS is an invasive tumor that responds poorly to treatment and for which there is yet no widely accepted effective therapy (9). Due to the fact that the vast majority of patients are at an advanced stage when they seek medical advice, the survival time is usually $<2$ years due to the poor response to chemotherapy (10). In recent years, it has been demonstrated that the use of radiotherapy and chemotherapy combined with autologous hematopoietic stem cell transplantation may improve treatment efficacy. In general, efficacy depends on a variety of factors, the most important being the location and stage of the tumor. For example, central nervous system and disseminated lesions are associated with an aggressive clinical course. The size of the tumor is also an important factor affecting the prognosis, and the survival time of patients with a primary tumor diameter of $>3.5 \mathrm{~cm}$ is short.
Despite the low incidence of HS, a number of cases (11-13) have been reported. Compared with previous reports, the tumor cells in the present case formed nests, with a cord-like distribution, which is generally not characteristic of HS, increasing the difficulty of morphological diagnosis. In addition, the tumor cells not only expressed the histiocytic antigens CD68 and lysozyme, but also expressed lymphocyte-associated antigens, such as CD4, CD5 and CD31, reflecting the complicated antigen expression pattern of HS. Unfortunately, our patient was unable to tolerate chemotherapy due to her poor physical condition; therefore, the patient underwent no intervention other than cervical lymph node biopsy and we were unable to collect more information on the treatment or outcome of HS, as she was lost to follow-up.

In conclusion, HS is a rare malignant tumor with poor prognosis. Due to its rapid invasiveness and high mortality rate, HS remains a medical challenge without effective treatment.

\section{Acknowledgements}

Not applicable.

\section{Competing interests}

The authors wish to declare that there is no known conflict of interests associated with this publication.

\section{Funding}

This study was supported by the Central South University Sports Medicine Scholarship and National College Students' Innovation and Entrepreneurship Training Program (grant no. 201710422116).

\section{Authors' contributions}

$\mathrm{JZ}$ and YL cared for the patient and contributed to the concept and design. JZ, WCW and FLX drafted the report.

\section{Ethics approval and consent to participate}

Not applicable.

\section{Availability of data and materials}

Not applicable.

\section{Consent for publication}

A signed written consent form was obtained from the patient regarding the publication of the case details and associated images.

\section{References}

1. Laviv Y, Zagzag D, Fichman-Horn S and Michowitz S: Primary central nervous system histiocytic sarcoma. Brain Tumor Pathol 30: 192-195, 2013.

2. Pollen M, El Jamal S, Lewin J and Manucha V: Histiocytic sarcoma in a kidney transplant patient: A case report and review of the literature. Case Rep Pathol 2016: 3591050, 2016. 
3. Hornick JL, Jaffe ES and Fletcher CD: Extranodal histiocytic sarcoma: Clinicopathologic analysis of 14 cases of a rare epithelioid malignancy. Am J Surg Pathol 28: 1133-1144, 2004

4. Yu L and Yang SJ: A case of primary histiocytic sarcoma arising from thyroid gland. Pathol Oncol Res 16: 127-132, 2010.

5. Swerdlow SH, Campo E, Pileri SA, Harris NL, Stein H, Siebert R, Advani R, Ghielmini M, Salles GA, Zelenetz AD, et al: The 2016 revision of the World Health Organization classification of lymphoid neoplasms. Blood 127: 2375-2390, 2016.

6. Takahashi E and Nakamura S: Histiocytic sarcoma: An updated literature review based on the 2008 WHO classification. J Clin Exp Hematop 53: 1-8, 2013.

7. Munoz J, Sanchez BE and Wang D: Histiocytic sarcoma of the thyroid. Am J Hematol 87: 531, 2012.

8. Pollen M, El Jamal S, Lewin J and Manucha V: Histiocytic Sarcoma in a Kidney Transplant Patient: A Case Report and Review of the Literature. Case Rep Pathol 2016: 3591050, 2016.
9. Shen XZ, Liu F, Ni RJ and Wang BY: Primary histiocytic sarcoma of the stomach: A case report with imaging findings. World J Gastroenterol 19: 422-425, 2013.

10. Zhao J, Niu X, Wang Z, Lu H, Lin X and Lu Q: Histiocytic sarcoma combined with acute monocytic leukemia: A case report. Diagn Pathol 10: 110, 2015.

11. Munoz J, Sanchez BE and Wang D: Histiocytic sarcoma of the thyroid. Am J Hematol 87: 531, 2012.

12. Pakravan A, Bhatia R, Oshima K, Chen G, Fesler M, Prather C and Taylor JR: Histiocytic sarcoma: The first reported case of primary esophageal involvement. Am J Gastroenterol 109: 291-292, 2014.

13. Nieuwenhuis MB, van der Salm SM, Verhoeff JJ, van der Kooi AJ, Slavujecvic-Letic I, Pals ST and Vos JMA: A 43-Year-Old Female with Multifocal Cerebral Lesions. Histiocytic Sarcoma. Brain Pathol 25: 371-372, 2015. 